\title{
Unusual Case of an Arterial Thoracic Outlet Syndrome due to Srb Anomaly
}

\author{
Saad Rustum ${ }^{1}$ Thomas Rodt ${ }^{2}$ Omke Teebken ${ }^{1}$ \\ 1 Department of Cardiothoracic, Transplantation, and Vascular \\ Surgery, Hannover Medical School, Hannover, Germany \\ 2 Institute for Radiology, Hannover Medical School, Hannover, \\ Germany
}

Claudia Schrimpf ${ }^{1}$ Thomas Aper $^{1} \quad$ Mathias Wilhelmi $^{1}$

Thorac Cardiovasc Surg Rep 2013;2:50-52.

\author{
Abstract \\ Keywords \\ - thoracic surgery \\ - anatomy \\ - artery/arteries \\ (includes all \\ peripheral arteries) \\ - thoracic outlet
}

A thoracic outlet syndrome (TOS) is caused by arterial or nervous obstruction because of skeletal or muscular anomalies and hypertrophies. Congenital rib anomalies occur with low incidences $(0.15-0.31 \%)$, predominantly affect the right side and are normally diagnosed at a young age or remain asymptomatic throughout life. Here, we report on the unusual case of a 71-year-old female patient with subacute ischemia of the left arm due to a TOS resulting from Srb anomaly, a very rare congenital rib anomaly.

\section{Case Report}

In this article, we report on the case of a 71-year-old female patient, who was presented with dry gangrene of the third and fifth finger of the left hand. History taking revealed that the symptoms had developed over a 3-week period before admission and were not associated with pain, trauma, arrhythmia, or temperature changes. A duplex ultrasound showed an arterial occlusion of the left subclavian and brachial artery. A computed tomographic scan, additionally, revealed a compression of the subclavian artery due to anomalous and hypertrophic first and second ribs, as described by Srb anomaly (-Figs. 1 and $\mathbf{2}$ ). Clinical and radiological findings led to the decision to proceed with urgent surgical intervention.

\section{Surgical Technique}

Using a left supraclavicular approach in supine position with a lightly lifted left shoulder, the patient's subclavian artery and vein were surgically exposed. The occluded artery was markedly shifted ventrally because of hypertrophic bony formations in terms of an exostosis of the first rib pushing from behind the artery. Following further arterial mobilization, partial exposure of the cervical plexus and intravenous application of 4,000 IU heparin, the subclavian artery was clamped and incised lengthwise. As anticipated, a thrombotic occlusion was found. Local thrombendarterectomy of the aneurysmatic subclavian artery was necessary because it was not possible to remove the old, organized thrombus. Direct closure of the artery was performed because of its enlargement and fragile wall.

Following surgical separation of the first and the second ribs, the hypertrophic segment (approximately $3 \mathrm{~cm}$ ) of the first rib was excised. Consequently, the subclavian artery was stress-relieved and free from any external compression.

Revascularization of the occluded left brachial artery was performed via a separate left cubital approach and in terms of a thrombolembolectomy using a Fogarty catheter. At the end of the entire procedure, the patient had strong radial and ulnar pulses.

Histopathological evaluation of the thrombus material and the resected rib confirmed the diagnosis of a chronic occlusion of the subclavian artery and revealed degenerative altered bone and cartilage material with focal remodeling received

March 12, 2013

accepted after revision

April 29, 2013

published online

June 17, 2013
DOI http://dx.doi.org/

$10.1055 / \mathrm{s}-0033-1347357$ ISSN 2194-7635. (c) 2013 Georg Thieme Verlag KG
Stuttgart · New York

License terms

$\Theta(1) \Theta \Theta$ 


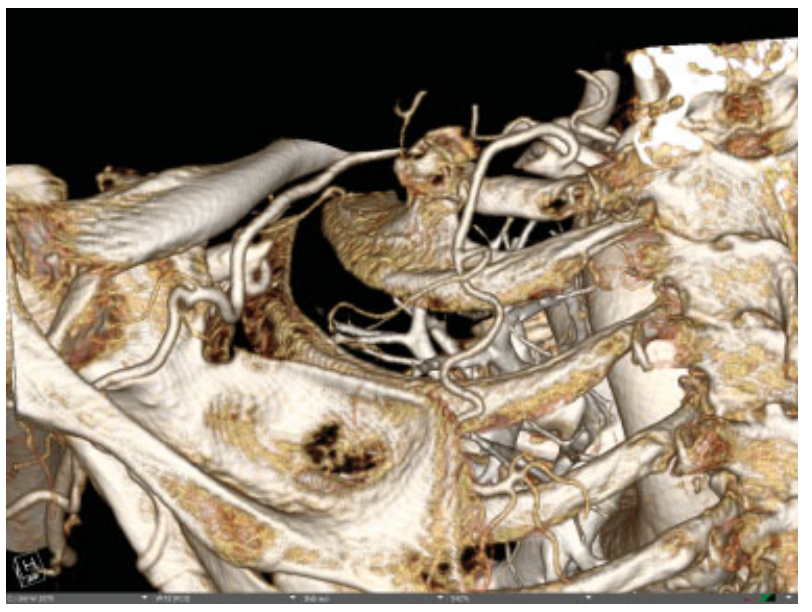

Fig. 1 Pre-op computed tomography: three-dimensional volume rendering from posterior, lateral, and superior view showing the hypertrophic region of the first rib as well as the partial fusion of the first and second rib. For better visualization of the relevant anatomical structures, the data volume was cropped.

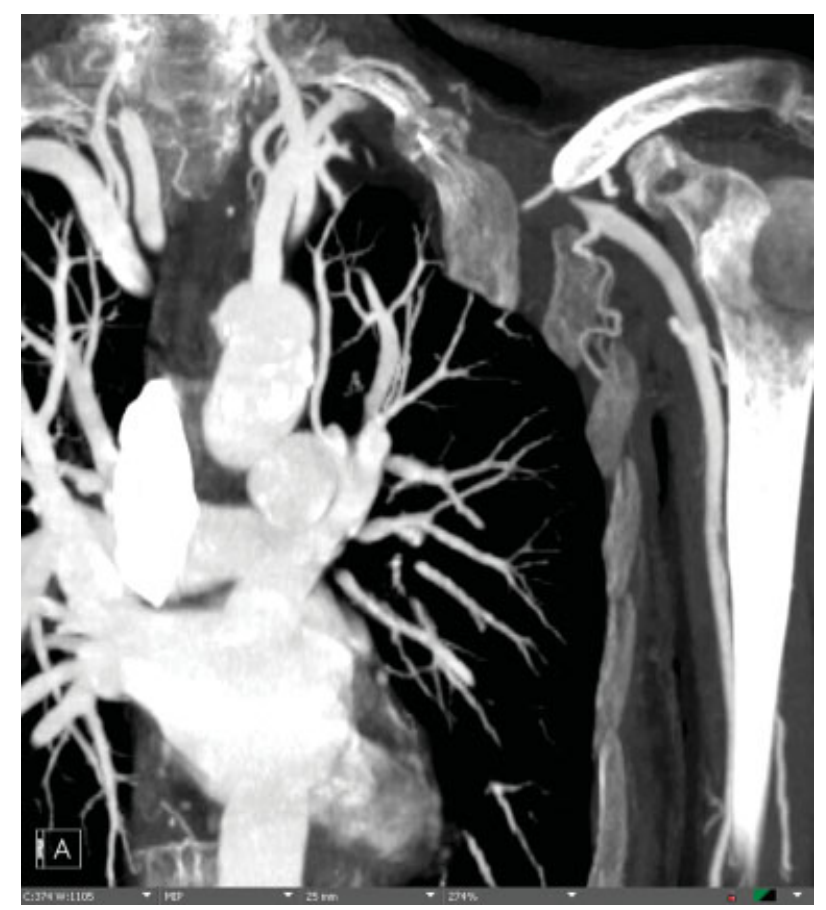

Fig. 2 Pre-OP computed tomography angiogram: coronal $25 \mathrm{~mm}$ maximum intensity projection slab showing the occlusion of the subclavian artery.

zones. The postoperative course was uneventful and the patient was referred to plastic surgery for finger amputation on postoperative day 4 .

\section{Discussion}

A thoracic outlet syndrome (TOS) is a constellation of upper extremity symptoms and indications resulting from compression of the neurovascular bundle in the thoracic outlet area. It is present in three different types according to the compressed structure as follows: neurogenic TOS, arterial TOS, and venous TOS. The latter is often also referred to as thoracic inlet syndrome. The pathophysiology and clinical symptoms of an arterial TOS are based on the effects of arterial obstruction due to compression by an anomalous first rib, a cervical rib, or a (hypertrophic) anterior scalene muscle. The incidence of hypertrophic ribs is around $0.76 \%$ and occurs equally in women and men. ${ }^{1}$ However, because of the fact that abnormalities of the (first) ribs are often overlooked on routine chest radiographs and most anomalous ribs remain asymptomatic throughout life, actual prevalence is potentially even higher. Clinically, the rib-induced compression may appear as arterial stenosis with or without associated poststenotic aneurysmal formation and accounts for $22 \%$ of all the arterial cases of the TOS. ${ }^{2}$ Most of the patients suffering from a TOS are young with a mean age of 37 years and similar incidence in men and women. Congenital rib anomalies occur with an incidence of between 0.15 and $0.31 \%$, tend to be slightly more frequent in women and, interestingly, are described as predominantly affecting the right side. ${ }^{3}$

The case presented here is very unusual for several of the following reasons: (i) TOS normally is diagnosed at younger age, (ii) the patient had no previous arterial or neurologic symptoms (iii) the patient suffered from a skeletal variant known as Srb anomaly, and (iv) rib anomalies most often affect the right side. Srb anomaly describes the seldom occurrence of partial or complete fusion of the first and second ribs, forming a solid bony plate with variable sternal articular patterns. ${ }^{4}$ Information on this anomaly is very scarce and there are extremely few reported cases in electronic databases. As a result, treating physicians may not take into consideration that a patient could be suffering from this rare anomaly, although knowledge of its occurrence could be essential for successful treatment and prophylaxis. Therefore, we would recommend that cross-sectional angiographic imaging modalities allowing for three-dimensional reconstruction, for example, computed tomography or magnetic resonance tomography should be conducted in all patients suffering from a TOS.

In conclusion, although Srb anomaly is a very rare clinical entity, cross-sectional imaging modalities should be initiated in all patients suffering from a TOS, as congenital anomalies can occur even in older and previously asymptomatic patients and complex rib anomalies are often overlooked in conventional radiography.

\section{Acknowledgment}

The authors thank Stanislav Kaschinski for the accurate elaboration of pre-op images, which ultimately led to the diagnosis of Srb anomaly.

\section{References}

1 Richard JS. Thoracic outlet syndrome: general considerations. In: Cambria, Cronenwett JL and Johnston KW, eds. Rutherford's vascular surgery, 7th ed., vol 2. Philadelphia, PA: Saunders; 2010:1865-1877 
52 Thoracic Outlet Syndrome due to Srb Anomaly Wilhelmi et al.

2 Stephen TS, Valentine RJ. Thoracic outlet syndrome: arterial. In: Cambria, Cronenwett JL and Johnston KW, eds. Rutherford's vascular surgery, 7th ed., vol 2. Philadelphia, PA: Saunders; 2010:1899-1906

3 Freyschmidt J. Shoulder Girdle and Thorax. In: Juergen Freyschmidt, Joachim Brossmann, Juergen Wiens, Andreas Sternberg, eds. Freyschmidt's “Koehler/ Zimmer” Borderlands of Normal and Early
Pathological Findings in Skeletal Radiography, 5th revised ed. New York, NY: Thieme; 2003:340-355

4 Gary MGuebert, Lindsay J Rowe, Terry R Yochum, Jeffrey R Thompson, and Chad J MaolaCongenital Anomalies and Normal Skeletal Variants. In: Terry R Youchum and Lindsay J Rowe eds. Essentials of skeletal radiology, 3rd ed., Vol 1. Philadelphia, PA: Lippincott Williams and Wilkins; 2005:257-404 